\title{
Investigation of a Novel S-1 Administration Schedule for Treating Metastatic and Recurrent Breast Cancer (KBCOG13)
}

\author{
MAYUKO MIKI ${ }^{1,2}$, SHINTARO TAKAO ${ }^{2}$, MUNEHARU KONISHI ${ }^{3}$, YASUSHI SHIGEOKA ${ }^{4}$, MASARU MIYASHITA ${ }^{5}$, \\ HIROFUMI SUWA ${ }^{6}$, YASUO MIYOSHI ${ }^{7}$, KOICHI HIROKAGA ${ }^{2}$, TOSHITAKA OKUNO ${ }^{8}$, KAZUHIKO YAMAGAMI ${ }^{9}$, \\ MICHIKO IMAMURA ${ }^{7}$, KEIKO MURASE ${ }^{7}$, AYAKO YANAI ${ }^{7}$ and HIROKAZU TANINO ${ }^{1}$ \\ ${ }^{1}$ Department of Breast Surgery, Kobe University Hospital, Kobe, Japan; \\ ${ }^{2}$ Department of Breast Surgery, Hyogo Cancer Center, Hyogo, Japan; \\ ${ }^{3}$ Department of Breast Surgery, Hyogo Prefectural Nishinomiya Hospital, Hyogo, Japan; \\ ${ }^{4}$ Department of Oncology, Yodogawa Christian Hospital, Osaka, Japan; \\ ${ }^{5}$ Department of Breast Surgery, Konan Hospital, Hyogo, Japan; \\ ${ }^{6}$ Department of Breast Surgery, Hyogo Prefectural Amagasaki Medical Center, Hyogo, Japan; \\ ${ }^{7}$ Department of Breast Surgery, Hyogo College of Medicine, Hyogo, Japan; \\ ${ }^{8}$ Department of Breast Surgery, Kobe City Nishi-kobe Medical Center, Kobe, Japan; \\ ${ }^{9}$ Department of Breast Surgery, Shinko Hospital, Kobe, Japan
}

\begin{abstract}
Background/Aim: S-1, a 5-fluorouracil(5-FU) oral anti-cancer drug, has been traditionally used with a schedule of 4-week oral administration followed by 2-week rest for breast cancer treatment. We, herein, aimed to investigate the clinical efficacy and safety of a schedule of 2-week oral administration followed by 1 -week rest for patients with metastatic breast cancer. Patients and Methods: We enrolled patients with HER2-negative metastatic breast cancer who had not received prior chemotherapy. $S-1$ was administered consecutively for 2-weeks followed by a 1-week rest. Results: Between September 1, 2013 and August 31, 2016, 32 patients were enrolled. The median follow-up time was 32.1 months. The median progression-free survival (PFS) was 9.4 months. Overall survival (OS) was 41.0 months, time to treatment failure (TTF) was 7.8 months, response rate $(R R)$ was $31.3 \%$, and disease control rate $(D C R)$ was $78.1 \%$. The incidence of grade 3 side-effects was not high. Conclusion: The 3-week schedule of S-1 can be considered useful as a treatment for patients with metastatic breast cancer, helping in maintaining a high quality of life.
\end{abstract}

This article is freely accessible online.

Correspondence to: Mayuko Miki, MD, Department of Breast Surgery, Kobe University Hospital, 7-5-2 Kusunokicho, Chuouku, Kobe City, Hyogo 650-0017, Japan. Tel: +81 783826273, Fax: +81 783826275, e-mail: mikimayu@med.kobe-u.ac.jp

Key Words: Metastatic breast cancer, 5-FU oral anti-cancer drug, S1, Tegafur, 3-week schedule.
In the treatment of metastatic breast cancer, chemotherapy that can maintain the quality of life is required (1). S-1, which was developed in Japan, is an oral anticancer drug that combines the prodrug of fluorouracil (5-FU), tegafur, with the 5-FU degradation inhibitor gimeracil and the phosphorylation inhibitor oteracil. It has been used to treat various cancers such as breast cancer, gastric cancer, head and neck cancer and lung cancer (2-4).

There are two clinical trials of S-1 for metastatic and recurrent breast cancer in Japan, with response rates of $42 \%$ and $40.7 \%$, respectively, that are comparable to taxanes (5). Among them, the response rate of patients who were not treated with chemotherapy for metastatic and recurrent cancer (59 out of 81 cases) was reported to be $49.2 \%$.

The S-1 Cooperative Study Group's Phase II study, which was a trial conducted for patients with recurrent breast cancer within 1 chemotherapy regimen, showed that the response rate was $41.7 \%$, and the clinical benefit rate was $45.3 \%(5)$.

The SELECT BC trial is a randomized, controlled, phase III trial that assesses overall survival for S-1 versus taxanes for patients with metastatic breast cancer (6). In this study, non-inferiority of S-1 was reported relative to taxanes with respect to overall survival (OS).

S-1 is used with a schedule of 4-week oral administration followed by a 2-week rest (6-week schedule) for breast cancer, but for lung cancer and head and neck cancer, reports have indicated that a 3-week schedule is good in terms of reducing side-effects, improving drug compliance and quality of life (79 ). The 3-week schedule may be better than the 6-week schedule in breast cancer as well, but there are few clinical 
trial data on 3-week schedule for breast cancer. Therefore, in this study, we examined the efficacy and safety of the 3-week schedule of S-1. The effects of S-1 are fully expected in the chemotherapy-naive cases that are the subject of this study.

\section{Patients and Methods}

Study design and patients. The patients in this study were recruited from 8 hospitals of the Kobe Breast Cancer Oncology Group (KBCOG) in Japan. Patient eligibility criteria for this study were as follows: breast cancer with histological confirmation, HER2negative or unknown, metastatic or recurrent breast cancer with at least one evaluable lesion, female gender over 20 years of age, and no previous chemotherapy for their recurrent or metastatic disease. Preoperative or postoperative adjuvant use of oral fluorouracil was allowed if at least 24 weeks had elapsed since the final day of treatment, Eastern Cooperative Oncology Group performance status of $0-2$, adequate organ function. leukocytes $\geq 3,000 / \mathrm{mm}^{3}$, neurocytes $\geq 1,500 / \mathrm{mm}^{3}$ hemoglobin $\geq 9.0 \mathrm{~g} / \mathrm{dl}$; platelets $100,000 / \mathrm{mm}^{3}$; serum total bilirubin $\leq 2.0 \mathrm{mg} / \mathrm{dl}$; aspartate aminotransferase (AST) and alanine aminotransferase (ALT) $<100$ IU/l; creatinine clearance rate $(\mathrm{Ccr}) 50 \mathrm{ml} / \mathrm{min}]$, normal cardiac function, and were from end of prior hormone therapy $>7$ days; prior radiation therapy $>14$ days. Patient exclusion criteria for this study were: if patients had a history of hypersensitivity to the protocol treatment drugs; had active secondary cancer; had brain metastases with any symptoms; had severe liver metastasis, lymphatic pulmonary metastasis, pleural effusion, ascites or pericardial effusions requiring emergency treatment; had active infections; had interstitial pneumonia or pulmonary fibrosis; were HBs antigen positive; had diabetes treated with insulin; had psychosis; and were pregnant women.

This study was performed in accordance with the ethical principles of the Declaration of Helsinki and approved by the institutional review board of all participating hospitals. Written informed consent was obtained from all patients prior to enrollment in this study.

Treatment schedule. S-1 was administered orally at 40-60 mg, twice daily after breakfast and dinner, consecutively for 2 weeks followed by 1 week of rest ( 1 course). The dose of S-1 was determined according to the body surface area (BSA) and creatinine clearance rate (Ccr) (Table I).

If administration could not be started, it could be postponed. If an adverse event occurred, administration was discontinued, and after recovery, the dose was reduced by one step and resumed. Treatment is discontinued if adverse events occur after the two-step dose reduction. If the doctor in charge judged it safe, the dose could be increased by one step. Treatment was discontinued if administration could not be initiated more than 28 days after the last day of administration.

Treatment was discontinued when the tumor progression or adverse events unable to be controlled by dose modification or temporary withdrawal of S-1. Treatment was also discontinued at patient request.

Study objectives. This efficacy was evaluated based on the Response Evaluation Criteria in Solid Tumors (RECIST) criteria, categorized into four categories: complete response (CR), partial response (PR),
Table I. Starting dose of $S-1$ and dose-reduction strategy of S-1. The dose of $S-1$ was determined according to the body surface area (BSA) and creatinine clearance rate $(\mathrm{Ccr})$.

Starting dose of S-1

\begin{tabular}{lcc}
\hline $\operatorname{BSA}\left(\mathrm{m}^{2}\right)$ & \multicolumn{2}{c}{ Creatinine clearance rate $(\mathrm{Ccr})$} \\
\cline { 2 - 3 } & $\geq 80 \mathrm{ml} / \mathrm{min}$ & $50 \mathrm{ml} / \mathrm{min} \leq \mathrm{x}<80 \mathrm{ml} / \mathrm{min}$ \\
\hline$\geq 1.5 \mathrm{~m}^{2}$ & $120 \mathrm{mg} /$ day & $100 \mathrm{mg} /$ day \\
$1.25 \mathrm{~m}^{2} \leq \mathrm{x}<1.5 \mathrm{~m}^{2}$ & $100 \mathrm{mg} /$ day & $80 \mathrm{mg} /$ day \\
$<1.25 \mathrm{~m}^{2}$ & $80 \mathrm{mg} /$ day & $60 \mathrm{mg} /$ day \\
\hline
\end{tabular}

Dose-reduction strategy of S-1

\begin{tabular}{lcc}
\hline Starting dose & First reduction & Second reduction \\
\hline $120 \mathrm{mg} /$ day & $100 \mathrm{mg} /$ day & $80 \mathrm{mg} /$ day \\
$100 \mathrm{mg} /$ day & $80 \mathrm{mg} /$ day & $60 \mathrm{mg} /$ day \\
$80 \mathrm{mg} /$ day & $60 \mathrm{mg} /$ day & $40 \mathrm{mg} /$ day \\
\hline
\end{tabular}

stable disease (SD), and progressive disease (PD). CR was defined as the clinical disappearance of all target lesions. PR was defined as a reduction of the sum of all measurable lesions by at least $30 \%$. PD was defined as an increase of the sum of all measurable lesions by more than $20 \%$, or as the appearance of a new lesion. Stable disease (SD) was not defined as CR, PR, or PD. Long SD was defined as SD lasting for more than 24 weeks.

We planned this study to verify the efficacy and toxicity of a 3week S-1 schedule as first-line chemotherapy for MBC. The primary endpoint was progression-free survival (PFS). Progression-free survival was defined as the time from the date of participation in this trial to the earliest date of disease progression or death from any cause. The secondary endpoints were overall survival (OS), time to treatment failure (TTF), overall response rate (ORR), disease control rate (DCR), and safety. TTF was defined as the interval between the date of participation in this trial and the earliest date of disease progression, withdrawal of study treatment for any reason, or death from any cause. ORR was defined as CR+PR.DCR was defined as $\mathrm{CR}+\mathrm{PR}+\mathrm{SD}$. Adverse events were assessed using the Common Terminology Criteria for Adverse Events v4.0 (CTCAE v4.0).

Statistical analysis. Although retrospective surveys after third-line treatment have been reported on S-1 metastatic or recurrent breast cancer, there are few prospective clinical trials. According to previous reports, the PFS (TTP) of S- 1 for patients who have been treated with anthracycline and taxane after the third line is about 2.5 to 6 months $(10-12)$.

The required sample size was estimated based on a threshold PFS of 3.5 months and an expected PFS of 6 months, a patient registration period of at least 2 years, a mean follow-up of 3 years, $90 \%$ power, and an alpha value of 0.05 (one-sided) using the SWOG One Arm Survival. The target sample size was determined to be at least 30 patients, so we recruited 35 participants. The Kaplan-Meier method is used to estimate the progression-free survival rate. Efficacy parameters were reported with two-sided $95 \%$ confidence intervals $(\mathrm{CI})$. 


\section{Results}

Patient characteristics. Between September 1, 2013, and August 31, 2016, we enrolled 32 patients. The median follow-up time was 32.1 months, and the range was 4.8-69.0 months. The demographic characteristics of the 32 patients are presented in Table II. Twenty-eight of the $32(87.5 \%)$ patients were hormone-receptor positive, and 4 of the 32 $(12.5 \%)$ patients were triple-negative. Fifteen patients received perioperative chemotherapy. Anthracycline, taxane, and 5 -FU were used in $43.8 \%, 40.6 \%$, and $25.0 \%$ of the patients, respectively. S-1 was the first-line chemotherapy for metastatic breast cancer in all patients.

Efficacy. Median PFS was 9.4 months (95\% CI=5.0-13.8) (Figure 1), exceeding the expected PFS 6 months. Median OS was 41.0 months (95\% CI=26.8-NA) (Figure 2), and median TTF was 7.6 months (95\% CI=2.7-11.5) (Figure 3). ORR was $31.3 \%$, and DCR was $78.1 \%$ (Table III).

Safety. Common toxicities were anemia, fatigue, anorexia, and nausea. Details are shown in Table IV. There were few Grade 3 toxicities. There was no elevation of AST or ALT. All adverse events were manageable.

Feasibility. The feasibility of S-1 is shown in Table V. Eight patients had creatinine clearance $<80 \mathrm{ml} / \mathrm{min}$. The $\mathrm{S}-1$ dose was reduced for each of the eight patients. Median number of treatment cycles (range) was $10(2-47)$. The reasons for interruption of treatment were 11 adverse events, 3 infections, and 1 to start radiation therapy. Twenty-six patients $(81.3 \%)$ discontinued due to progressive disease and 5 patients (15.6\%) discontinued due to adverse events. The other patients were discontinued for treatment of other diseases. PD consisted of 14 cases of exacerbation of existing lesions, 17 cases of new lesions, and 1 case judged by the attending physician due to elevated tumor markers. The reason for discontinuation of AEs was anemia, infection, nausea, decreased vision, and myalgia in 1 patient each. Relative dose intensity (RDI) was $94.5 \%$. RDI was calculated with the starting dose at $100 \%$.

\section{Discussion}

Two clinical trials have evaluated the efficacy and safety of S-1 for breast cancer. In the Phase II study of the S-1 Cooperative Study Group, the efficacy and safety of S-1 were evaluated in patients with metastatic breast cancer who had received 1 chemotherapy regimen. In this clinical study, RR was $41.7 \%$ and CBR $45.3 \%$. The median survival time was 872 days (5). The SELECT-BC study examined the noninferiority of overall survival of S-1 to taxane and the superiority of health-related quality of life in the first-line treatment for metastatic breast cancer (6). OS of the S-1
Table II. Patient characteristics $(n=32)$.

\begin{tabular}{|c|c|}
\hline & $\mathrm{n}(\%)$ \\
\hline Age (range) & $64(38-80)$ \\
\hline \multicolumn{2}{|l|}{ Menopausal status } \\
\hline Premenopausal & $7(21.9 \%)$ \\
\hline Postmenopausal & $25(88.1 \%)$ \\
\hline \multicolumn{2}{|l|}{ Performance Status (ECOG) } \\
\hline 0 & $23(71.9 \%)$ \\
\hline 1 & $7(21.9 \%)$ \\
\hline 2 & $2(6.3 \%)$ \\
\hline \multicolumn{2}{|l|}{ Metastatic or Recurrence } \\
\hline Metastatic & $9(28.1 \%)$ \\
\hline Recurrence & $23(71.9 \%)$ \\
\hline \multicolumn{2}{|l|}{ Metastatic site (overlaps) } \\
\hline Local & $8(25 \%)$ \\
\hline Lymph nodes & $14(43.8 \%)$ \\
\hline Lung & $13(40.6 \%)$ \\
\hline Bone & $22(68.6 \%)$ \\
\hline Liver & $12(37.5 \%)$ \\
\hline Contralateral breast & $2(6.3 \%)$ \\
\hline \multicolumn{2}{|l|}{ Hormonal receptor } \\
\hline ER+ and/or PgR+/HER2- & $28(87.5 \%)$ \\
\hline ER- and PgR-/HER2- & $4(12.5 \%)$ \\
\hline \multicolumn{2}{|l|}{ Pathology } \\
\hline Invasive ductal carcinoma & $28(87.5 \%)$ \\
\hline Invasive lobular carcinoma & $2(6.3 \%)$ \\
\hline Others & $2(6.3 \%)$ \\
\hline \multicolumn{2}{|l|}{ Neo/adjuvant chemotherapy } \\
\hline Yes & $15(46.9 \%)$ \\
\hline Anthracycline & $14(43.8 \%)$ \\
\hline Taxane & $13(40.6 \%)$ \\
\hline 5-FU & $8(25 \%)$ \\
\hline No & $16(50 \%)$ \\
\hline Unknown & $1(3.1 \%)$ \\
\hline \multicolumn{2}{|l|}{ Adjuvant endocrine therapy for } \\
\hline $\mathrm{ER}+$ recurrent breast cancer & $\mathrm{n}=20$ \\
\hline Yes & $19(95 \%)$ \\
\hline Unknown & $1(5 \%)$ \\
\hline Endocrine therapy for MBC & $\mathrm{n}=28$ \\
\hline Yes & $26(93 \%)$ \\
\hline No & $2(7 \%)$ \\
\hline Bone-modifying agents & $19(59.4 \%)$ \\
\hline
\end{tabular}

ER: Estrogen receptor; PgR: progesterone receptor; HER2: human epidermal growth factor receptor 2; ECOG: Eastern Cooperative Oncology Group; MBC:Metastatic and recurrent breast cancer.

group and the taxane group were 35.0 months vs. 37.2 months $(p=0.015)$, and PFS were 9.6 months vs. 11.0 months. Non-inferiority of OS of S-1 to taxane was demonstrated. The superiority of health-related quality of life was also shown. S-1 was used with a 6-week schedule in both studies.

This study investigated the efficacy and safety of a 3-week S-1 schedule as a first-line treatment for metastatic breast cancer. The PFS of this study was 9.4 months, which was longer than the expected value at 6 months. The RR was $31.3 \%$, CBR was $40.6 \%$, and RR was slightly lower than that 


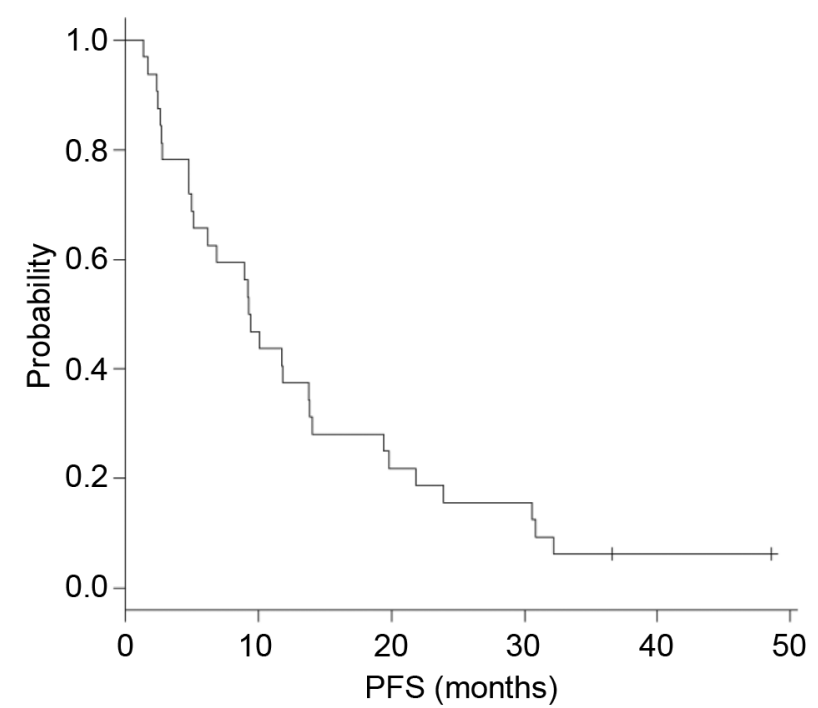

Figure 1. Kaplan-Meier estimates of progression-free survival $(P F S)$ in eligible patients $(n=32)$. Median PFS was 9.4 months (95\% CI=5.0-13.8).

Table III. Response rate in eligible patients $(n=32)$. This efficacy of $S$ 1 was evaluated based on the Response Evaluation Criteria in Solid Tumors (RECIST) criteria.

\begin{tabular}{lc}
\hline & $\mathrm{n}(\%)$ \\
\hline Total response & \\
Complete response (CR) & 0 \\
Partial response (PR) & $10(31.3 \%)$ \\
Stable disease (SD) & $12(37.5 \%)$ \\
Long SD (SD $>24 \mathrm{w})$ & $3(9.4 \%)$ \\
Progressive disease (PD) & $6(18.8 \%)$ \\
Not evaluable (NE) & $1(3.1 \%)$ \\
Overall response rate (CR+PR) & $31.3 \%$ \\
Clinical benefit rate (CR+PR+long SD) & $40.6 \%$ \\
Disease control rate (CR+PR+SD) & $78.1 \%$ \\
\hline
\end{tabular}

reported by the S-1 Cooperative Study Group (5). However, in this study, about half of the 15 cases had SD, and DCR was $78.1 \%$. The results of this test were not inferior to those of the previously reported test of the 6-week schedule.

Five patients $(15.6 \%)$ stopped S-1 due to side-effects. One patient discontinued treatment due to a grade 3 side-effect on her eyes. The eye disorder caused by S-1 has not yet received much attention in the treatment of breast cancer. However, reports indicate the eye disorder associated with $\mathrm{S}-1$ is a side-effect for $16-25.3 \%$ of patients $(13,14)$. Termination of $\mathrm{S}-1$ eliminates the eye symptoms, so it is important to notice the eye disorder early.

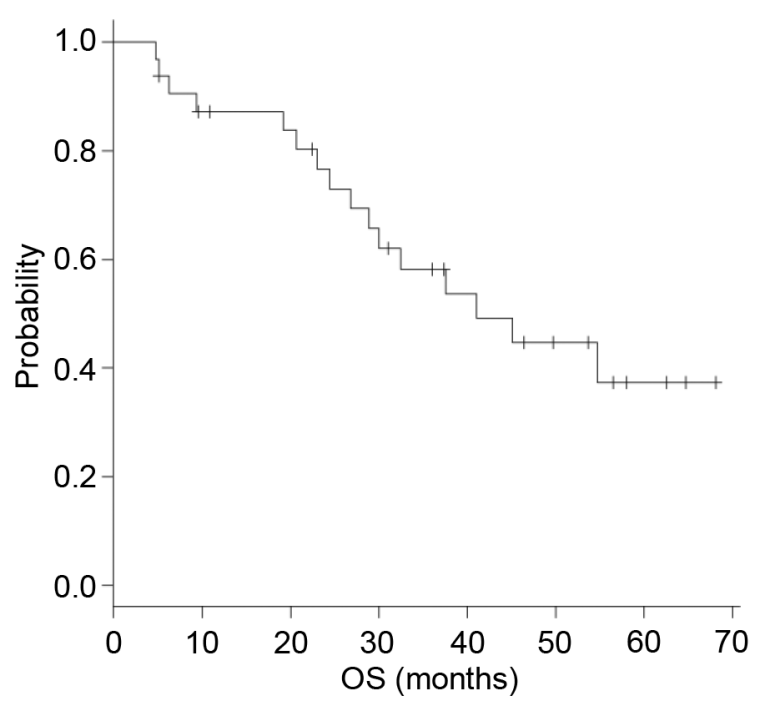

Figure 2. Kaplan-Meier estimates of overall survival (OS) in eligible patients $(n=32)$. Median OS was 41.0 months (95\% CI=26.8-NA).

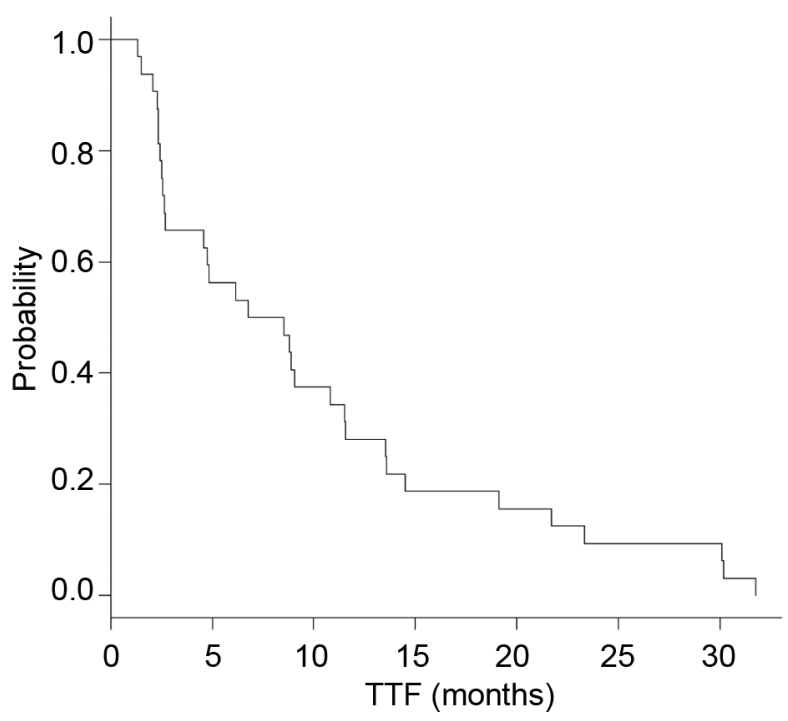

Figure 3. Kaplan-Meier estimates of time to failure (TTF) in eligible patients $(n=32)$. Median TTF was 7.6 months $(95 \% C I=2.7-11)$.

In addition, treatment was discontinued at the request of patients with grade 2 anemia, infection, nausea, and myalgia. All side-effects disappeared after discontinuation. Of the 27 patients who were able to continue treatment, eight patients had reduced their doses of S-1 due to side-effects. Discontinuation due to side-effects was judged an event in the evaluation of TTF. However, in the evaluation of PFS, discontinuation of S-1 was not judged as an event, but the 
Table IV. Hematological and non-hematologic adverse events $(n=32)$. Numbers are patients who reported events. Severity was graded according to the Common Terminology Criteria for Adverse Events v4.0 (CTCAE v4.0).

\begin{tabular}{|c|c|c|c|c|}
\hline & Grade $1(\%)$ & Grade $2(\%)$ & Grade $3(\%)$ & Any $(\%)$ \\
\hline \multicolumn{5}{|l|}{ Hematological } \\
\hline Leukopenia & $5(15.6 \%)$ & $8(25.0 \%)$ & $1(3.1 \%)$ & $14(43.8 \%)$ \\
\hline Neutropenia & $2(6.3 \%)$ & $4(12.5 \%)$ & $3(9.4 \%)$ & $9(28.1 \%)$ \\
\hline Anemia & $15(46.9 \%)$ & $4(12.5 \%)$ & & $19(59.4 \%)$ \\
\hline Thrombocytopenia & $11(34.4 \%)$ & & & $11(34.4 \%)$ \\
\hline AST increasing & $11(34.4 \%)$ & $2(6.3 \%)$ & & $13(40.6 \%)$ \\
\hline ALT increasing & $11(34.4 \%)$ & $1(3.1 \%)$ & & $12(37.5 \%)$ \\
\hline Bilirubin & $8(25.0 \%)$ & $1(3.1 \%)$ & $1(3.1 \%)$ & $11(34.4 \%)$ \\
\hline Creatinine & $8(25.0 \%)$ & & & $8(25.0 \%)$ \\
\hline \multicolumn{5}{|l|}{ Non-hematological } \\
\hline Diarrhea & $10(31.3 \%)$ & $1(3.1 \%)$ & & $11(34.4 \%)$ \\
\hline Mucositis & $9(28.1 \%)$ & $4(12.5 \%)$ & & $13(40.6 \%)$ \\
\hline Rash & $9(28.1 \%)$ & $2(6.3 \%)$ & & $11(34.4 \%)$ \\
\hline Nausea & $12(37.5 \%)$ & $3(9.4 \%)$ & & $15(46.9 \%)$ \\
\hline Vomiting & $4(12.5 \%)$ & $1(3.1 \%)$ & & $5(15.6 \%)$ \\
\hline Fatigue & $15(46.9 \%)$ & $2(6.3 \%)$ & & $17(53.1 \%)$ \\
\hline Anorexia & $10(31.3 \%)$ & $3(9.4 \%)$ & $1(3.1 \%)$ & $14(43.8 \%)$ \\
\hline Nosebleed & $2(6.3 \%)$ & & & $2(6.3 \%)$ \\
\hline Dysgeusia & $5(15.6 \%)$ & $1(3.1 \%)$ & & $6(18.8 \%)$ \\
\hline Eye disorder & $9(28.1 \%)$ & $1(3.1 \%)$ & $1(3.1 \%)$ & $11(34.4 \%)$ \\
\hline Abdominal pain & $1(3.1 \%)$ & & & $1(3.1 \%)$ \\
\hline Pigmentation & $6(18.8 \%)$ & & & $6(18.8 \%)$ \\
\hline Constipation & $5(15.6 \%)$ & & & $5(15.6 \%)$ \\
\hline Hand-foot syndrome & $4(12.5 \%)$ & $3(9.4 \%)$ & & $7(21.9 \%)$ \\
\hline Sensory neuropathy & $2(6.3 \%)$ & & & $2(6.3 \%)$ \\
\hline
\end{tabular}

PD of the next treatment was judged as an event. Therefore, TTF was 7.8 months, but PFS was 9.6 months. Even so, TTF was longer than the expected PFS of 6 months.

In other carcinomas, the effects, side-effects, and compliance have been reported on the 3 -week schedule and the 6-week schedule. In the treatment of head and neck cancer and oral cancer, it has been reported that the 3-week schedule tends to have fewer grade 3 and 4 side-effects than the 6 -week schedule $(9,15)$. In the clinical trial of breast cancer using S-1 at a 6-week schedule, AST and ALT elevation was reported at $37 \%$ and $29 \%$ in all grades and $3 \%$ for both AST and ALT in grade 3 (12). In this study, grade 3 side-effects were minimal, and grade 3 or 4 AST and ALT elevation was not observed.

In the treatment of head and neck cancer, the 3-week S-1 schedule was reported with better medication compliance than the 6-week schedule (9). The cumulative rates of the relative total administration dose at $100 \%$ were $54.9 \%(95 \%$ $\mathrm{CI}=40.1-69.7 \%)$ in the 3 -week schedule and $34.3 \%(95 \%$ $\mathrm{CI}=21.1-47.4 \%)$ in the 6-week schedule. In two clinical trials of lung cancer, $20 \%$ and $31 \%$ patients discontinued treatment due to side-effects $(7,8)$. In our study, 5 patients $(15.6 \%)$ discontinued due to adverse events, so the completion rate in this study was $84.4 \%$.
Table V. The feasibility of $S-1$ in eligible patients $(n=32)$.

\begin{tabular}{lc}
\hline & $\mathrm{n}(\%)$ \\
\hline Starting dose & \\
$100 \%$ & $24(75 \%)$ \\
Reduced dose & $8(25 \%)$ \\
Median number of treatment cycles (range) & $10(2-47)$ \\
Dose reduction & \\
Yes & $8(25 \%)$ \\
No & $24(75 \%)$ \\
Interruption & \\
Yes & $16(50 \%)$ \\
No & $16(50 \%)$ \\
Reason of discontinuation & \\
Progressive disease & $26(81.3 \%)$ \\
Adverse event & $5(15.6 \%)$ \\
Other & $1(3.1 \%)$ \\
Relative dose intensity (mean) & $94.5 \%$ \\
95\% CI & $91.2-97.9$ \\
\hline
\end{tabular}

Reports indicate that some patients discontinued treatment due to side-effects appearing from the third week in the 6-week schedule (9). In the 3 -week schedule, patients have a treatment break in the third week. The S-1 side-effects then do not 
become worse. A better schedule makes better compliance and causes better response. SELECT BC was performed on the 6week schedule, but the 3-week schedule may reduce the severe side-effects of S-1 and improve the results.

Although new molecularly targeted drugs, immunotherapies, and other treatments have been developed, these treatments have side-effects such as autoimmune diseases. In addition, the new treatment options are expensive. A new treatment that is effective with minimal side-effects, such as the S-1 three-week method, would contribute to both the patient and the medical economy.

\section{Conflicts of Interest}

The Authors declare no competing financial interests.

\section{Authors' Contributions}

M.M, and S.T designed trial, M.M, and H.T wrote the paper; other Authors, except H.T, collected patients' data.

\section{Acknowledgements}

We greatly appreciate all the women who participated in this trial and all investigators who were dedicated to this study. We also give special thanks to Prof. Kohei Akazawa for statistical analysis support and Dr. Norio Kohno, the founder of Kobe Breast Cancer Oncology Group (KBCOG).

\section{References}

1 Hortobagyi GN: Treatment of breast cancer. N Engl J Med 339(14): 974-984, 1998. PMID: 9753714. DOI: 10.1056/ NEJM199810013391407

2 Fujii M, Tomita K, Nishijima W, Tsukuda M, Hasegawa Y, Ishitoya J, Yamane H, Homma A and Tomita T: Phase I/II study of s-1 plus cisplatin combination chemotherapy in patients with advanced/recurrent head and neck cancer. Jpn J Clin Oncol 40(3): 214-221, 2010. PMID: 19934112. DOI: 10.1093/jjco/hyp153

3 Nagashima F, Ohtsu A, Yoshida S and Ito K: Japanese nationwide post-marketing survey of $\mathrm{S}-1$ in patients with advanced gastric cancer. Gastric Cancer 8(1): 6-11, 2005. PMID: 15747168. DOI: 10.1007/s10120-004-0306-3

4 Okamoto I, Yoshioka H, Morita S, Ando M, Takeda K, Seto T, Yamamoto N, Saka H, Asami K, Hirashima T, Kudoh S, Satouchi M, Ikeda N, Iwamoto Y, Sawa T, Miyazaki M, Tamura K, Kurata T, Fukuoka M and Nakagawa K: Phase III trial comparing oral S-1 plus carboplatin with paclitaxel plus carboplatin in chemotherapy-naïve patients with advanced nonsmall-cell lung cancer: results of a west Japan oncology group study. J Clin Oncol 28(36): 5240-5246, 2010. PMID: 21079147. DOI: $10.1200 / \mathrm{JCO} .2010 .31 .0326$

5 Saek T, Takashima S, Sano M, Horikoshi N, Miura S, Shimizu S, Morimoto K, Kimura M, Aoyama H, Ota J, Noguchi S and Taguchi T: A phase II study of S-1 in patients with metastatic breast cancer - a Japanese trial by the S-1 Cooperative Study Group, Breast Cancer Working Group. Breast Cancer 11(2): 194202, 2004. PMID: 15550867. DOI: 10.1007/BF02968301
6 Takashima T, Mukai H, Hara F, Matsubara N, Saito T, Takano T, Park Y, Toyama T, Hozumi Y, Tsurutani J, Imoto S, Watanabe T, Sagara Y, Nishimura R, Shimozuma K, Ohashi Y and SELECT BC Study Group: Taxanes versus S-1 as the first-line chemotherapy for metastatic breast cancer (SELECT BC): an open-label, noninferiority, randomised phase 3 trial. Lancet Oncol 17(1): 90-98, 2016. PMID: 26617202. DOI: 10.1016/S1470-2045(15)00411-8

7 Nishiyama O, Taniguchi H, Kondoh Y, Takada K, Baba K, Saito H, Sugino Y, Yamamoto M, Ogasawara T, Kondo M, Imaizumi K, Hasegawa Y, Suzuki R, Shimokata K and Central Japan Lung Study Group: Phase II study of S-1 monotherapy as a first-line treatment for elderly patients with advanced nonsmall-cell lung cancer: the Central Japan Lung Study Group trial 0404. Anticancer Drugs 22(8): 811-816, 2011. PMID: 21317767. DOI: 10.1097/CAD.0b013e3283440231

8 Goto H, Okano Y, Machida H, Hatakeyama N, Ogushi F, Haku T, Kanematsu T, Urata T, Kakiuchi S, Hanibuchi M, Sone S and Nishioka Y: Phase II study of tailored S-1 monotherapy with a 1week interval after a 2-week dosing period in elderly patients with advanced non-small cell lung cancer. Respir Investig 56(1): 8086, 2018. PMID: 29325686. DOI: 10.1016/j.resinv.2017.09.003

9 Tsukuda M, Kida A, Fujii M, Kono N, Yoshihara T, Hasegawa Y, Sugita M and Chemotherapy Study Group of Head and Neck Cancer: Randomized scheduling feasibility study of S-1 for adjuvant chemotherapy in advanced head and neck cancer. Br J Cancer 93(8): 884-889, 2005. PMID: 16189518. DOI: 10.1038/sj.bjc.6602804

10 Fujii K, Kosaka J, Mouri Y, Yoshida M, Yorozuya K, Nakano S, Akizuki M, Fukutomi T and Kobayashi K: [Chemotherapy of a 2-week S-1 administration followed by 1-week rest for advanced and metastatic breast cancer]. Gan To Kagaku Ryoho 38(9): 1467-1470, 2011. PMID: 21918343.

11 Shien T, Shimizu C, Akashi-Tanaka S, Yonemori K, Kohno T, Hojo T, Ando M, Katsumata N, Kinoshita T and Fujiwara Y: Clinical efficacy of S-1 in pretreated metastatic breast cancer patients. Jpn J Clin Oncol 38(3): 172-175, 2008. PMID: 18296424. DOI: $10.1093 / \mathrm{jjco} / \mathrm{hyn} 001$

12 Ito S, Ohashi Y and Sasako M: Survival after recurrence in patients with gastric cancer who receive S-1 adjuvant chemotherapy: exploratory analysis of the ACTS-GC trial. BMC Cancer 18(1): 449, 2018. PMID: 29678146. DOI: 10.1186/s12885-018-4341-6

13 Koizumi W, Narahara H, Hara T, Takagane A, Akiya T, Takagi M, Miyashita K, Nishizaki T, Kobayashi O, Takiyama W, Toh Y, Nagaie T, Takagi S, Yamamura Y, Yanaoka K, Orita H and Takeuchi M: S-1 plus cisplatin versus S-1 alone for first-line treatment of advanced gastric cancer (SPIRITS trial): a phase III trial. Lancet Oncol 9(3): 215-221, 2008. PMID: 18282805. DOI: 10.1016/S1470-2045(08)70035-4

14 Tabuse H, Kashiwagi H, Hamauchi S, Tsushima T, Todaka A, Yokota T, Machida N, Yamazaki K, Fukutomi A, Onozawa Y, Mori K, Boku N, Ichinose M and Yasui H: Excessive watering eyes in gastric cancer patients receiving S-1 chemotherapy. Gastric Cancer 19(3): 894-901, 2016. PMID: 26362271. DOI: 10.1007/s10120-015-0540-X

15 Harada K, Ferdous $\mathrm{T}$ and Yoshida H: Investigation of optimal schedule of concurrent radiotherapy with S-1 for oral squamous cell carcinoma. Oncol Rep 18(5): 1077-1083, 2007. PMID: 17914556.

Received April 19, 2021

Revised May 19, 2021

Accepted May 20, 2021 